\title{
Heart rate response to non-signal tones'
}

WILLIAM G. CHASE AND FRANCES K. GRAHAM

UNIVERSITY OF WISCONSIN

The heart rate response to onset of 18-sec, nonsignal tones heard over $71 \mathrm{~dB}$ white noise was solely decelerative and habituated rapidly; the response to tone offset was similar to the onset response. It was suggested that cardiac deceleration is a component of the orienting reflex.

Sokolov (1963) has described two important reflex systems, orienting (OR) and defense, which serve respectively to increase or decrease sensitivity to stimulation. Although these reflexes involve many of the same autonomic and central changes and can not be differentiated by such responses as GSR and alpha-blocking, Sokolov concluded that they do differ in the direction of cephalic vasomotor response. $\mathrm{He}$ proposed that cephalic vasodilation, is a component of the OR, since it is evoked by stimulus change and habituates rapidly to nonsignal stimuli, and that vasoconstriction is a component of the defense reflex since it occurs with intense stimulation and shows little habituation.

Using these same criteria, Graham \& Clifton (1966) suggested that heart rate (HR) deceleration is also a component of the OR. This suggestion appeared to be contradicted by reports that nonsignal tones elicit a diphasic HR response of initial acceleration followed by a longer latency decleration. However, it was noted that the acceleratory phase was more prominent with stronger stimuli and habituated slowly if at all. Consequently, this initial acceleration met the criteria for a defensive startle reflex.

The present experiment tested whether a solely decelerative response would occur if nonsignal tones were presented over background noise. White noise of $71 \mathrm{~dB}$ produced a moderately intense level of stimulation but the changes in level were small and presumably should not elicit defense or startle reflexes. To determine whether the resulting response was an OR, two criteria were employed. First, since stimulus change is the effective stimulus for eliciting an $O R$, both increase and decrease of stimulus intensity may evoke it and response to stimulus offset should therefore resemble the response to onset. Second, there should be habituation across trials. The study also attempted to vary stimulus novelty by presenting one series of 10 identical tones and another series of 10 tones differing in frequency and intensity.

Method

Ten male university students served as Ss; eight were paid and two were given class credit. Half the Ss received the series of Identical tones followed by the series of different tones while the other half recelved the two series in reverse order. Stimuli were 10 tones equally spaced from 300 to $2000 \mathrm{cps}$ on a logarithmic scale and ranging from 60 to $87 \mathrm{~dB}$ re .002 microbars. A different tone was presented to each S 10 times during the identical-tone series; during the different-tone series, each tone was presented once with order balanced across Ss with a $10 \times 10$ Latin square.

Tones and continuous $71 \mathrm{~dB}$ white noise were presented via speakers in a sound-attenuated chamber while the electrocardiogram (EKG) was recorded. Other apparatus was located outside the chamber; details of recording and data processing are described in Chase, Graham, \& Graham (in press). After $S$ was instructed to sit quietly and keep muscle movements to a minimum, white noise was started and the first tone followed $1 \mathrm{~min}$ later. Tones were $18 \mathrm{sec}$ long with an interstimulus interval (offset to onset) averaging $60 \mathrm{sec}$ and randomly spaced within 45-75 sec. All Ss received the same sequence of intervals. At the end of the first series, there was a 2-min rest. White noise was left on and S remained seated but could stretch. After the rest, instructions not to move were repeated and the second series was presented 1 min later.

\section{Resulis and Discussion}

Heart rate, averaged over Ss, trials, and tones, is shown in Fig. 1 for the first sec preceding stimulus onset and for $28 \mathrm{sec}$ following onset. Response to both onset and offset appears to be a sharp deceleration, beginning within $1 \mathrm{sec}$ of stimulus change. The HR remains slightly depressed from prestimulus level throughout stimulation, but recovers after the offset response.

For purposes of analysis, the data were grouped into three trial-blocks of three trials each, omit-

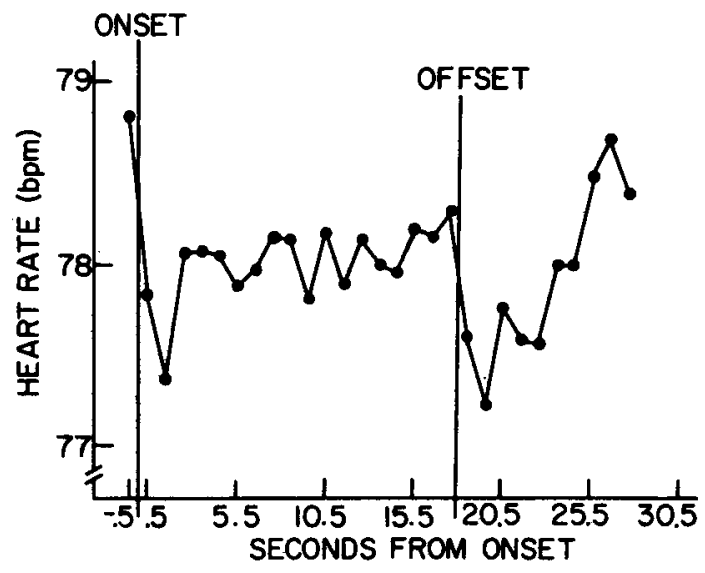

Fig. 1. Second-by-second HR to 18-sec tones. Data are averaged over Ss and trials and are plotted at midpoints of each interval. 


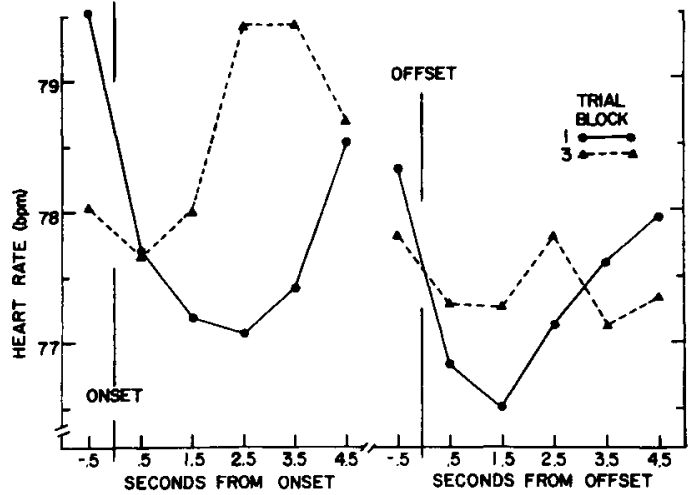

Fig. 2. Habituation in onset and offset responses to 18-sec tones.

ting the seventh trial. Trend analyses were performed across four successive 6-sec periods: (1) the prestimulus sec and five post-onset sec, (2) post-onset sec $6-11$, (3) post-onset sec 12-17, (4) the first sec preceding offset and the $5 \mathrm{sec}$ following offset.

No trends across sec or changes in trend across trial-blocks approached significance for the second and third periods, indicating a stable HR during sec 6-17. Trends for the first and fourth periods, however, provided statistical support for the onset and offset responses. Quadratic and Cubic components together accounted for $84 \%$ of the seconds variance during the onset period (Quadratic $F=15.65$, df $=$ 1/8, error $M S=6.17, p<.01$; Cubic $F=7.00, \mathrm{df}=1 / 8$, error $M S=10.47, p<.05)$. Offset trends were less pronounced, with only the Quadratic component approaching significance $(\mathrm{p}<.10)$.

Figure 2 illustrates rapid habituation of the decelerative response at both onset and offset; very little deceleration remained by the last three trials. Habituation of the onset response was confirmed by changes in trend over trial-blocks (Trial-blocks by Linear Seconds $F=11.25, \mathrm{df}=2 / 16$, error $\mathrm{MS}=9.10, p<.0025$; Trial-blocks by Quadratic Seconds $F=4.43, \mathrm{df}=2 / 16$, error $\mathrm{MS}=18.6, \mathrm{p}<.05)$. The maximum HR decreases from prestimulus in Trial-blocks 1 and 2 were also significant $(t=3.40$ and $2.77, d f=59, p<.01)$, but neither the maximum decrease nor the maximum increase was significant in the third trial-block. Again, changes in trend at offset were less pronounced (Trial-blocks by Quadratic Seconds $F=3.44$, df $=2 / 16$, error $M S=$ $9.89, \mathrm{p}<.10)$. However, the significant maximum HR decrease from prestimulus in the first trial-block $(t=3.08$, $d f=59, p<.01)$ and the absence of any significant change on subsequent trial-blocks suggest rapid habituation.

No period showed significant trends in response either to the first and second tone series or in response to identical and different tones. Presumably, spontaneous recovery of the habituated OR occurred during rest since some degree of recovery is a common finding. Failure to obtain slower habituation with variation of tones is the only result not consistent with expectation. It is possible that this manipulation did not produce sufficient novelty.

The present study demonstrates that $\mathrm{HR}$ response to nonsignal tones does not necessarily show an initial accelerative component and suggests that relative stimulus intensity is as important as absolute level in determining the form of the HR response to nonsignal stimuli. Although background level was not discussed by Sokolov, it may be a critical parameter in determining whether a given stimulus intensity elicits an OR or defense reflex.

The study also supports the idea that HR decleration is a component of the OR. With a long stimulus duration, the same response occurred at both onset and offset and both responses habituated rapidly. These criteria differentiate the oR from the defense reflex. Cardiac deceleration, therefore, meets several important criteria which identify the OR.

References

CHASE, W. G., GRAHAM, F. K., \& GRAHAM, D. T. Components of heart rate response in anticipation of reaction time and exercise tasks. J. exp. Psychol, in press.

GRAHAM, F. K., \& CLIFTON, R. K. Heart-rate change as a component of the orienting response. Psychol Bull, 1966, 65, 305-320.

SOKOLOV, E. N. Perception and the conditioned reflex. New York: Macmillan, 1963.

Note

1. This research was supported by grants HD01490 and K3-MH-21762 from the National Institutes of Health. Computer services were provided through grant FR000249 to the Biomedical Computing Division and an NSF grant through the University of Wisconsin Research Committee. 